\title{
Stress leads to aberrant hippocampal involvement when processing schema-related information
}

\author{
Susanne Vogel, ${ }^{1}$ Lisa Marieke Kluen, ${ }^{1}$ Guillén Fernández, ${ }^{2}$ and Lars Schwabe ${ }^{1}$ \\ ${ }^{1}$ Department of Cognitive Psychology, Institute of Psychology, University of Hamburg, 20146 Hamburg, Germany; ${ }^{2}$ Donders Institute \\ for Brain, Cognition and Behaviour, Radboud University Medical Center, 6525 EN Nijmegen, The Netherlands
}

\begin{abstract}
Prior knowledge, represented as a mental schema, has critical impact on how we organize, interpret, and process incoming information. Recent findings indicate that the use of an existing schema is coordinated by the medial prefrontal cortex ( $\mathrm{mPFC}$ ), communicating with parietal areas. The hippocampus, however, is crucial for encoding schema-unrelated information but not for schema-related information. A recent study indicated that stress mediators may affect schema-related memory, but the underlying neural mechanisms are currently unknown. Here, we thus tested the impact of acute stress on neural processing of schema-related information. We exposed healthy participants to a stress or control manipulation before they processed, in the MRI scanner, words related or unrelated to a preexisting schema activated by a specific cue. Participants' memory for the presented material was tested 3-5 d after encoding. Overall, the processing of schema-related information activated the mPFC, the precuneus, and the angular gyrus. Stress resulted in aberrant hippocampal activity and connectivity while participants processed schema-related information. This aberrant engagement of the hippocampus was linked to altered subsequent memory. These findings suggest that stress may interfere with the efficient use of prior knowledge during encoding and may have important practical implications, in particular for educational settings.
\end{abstract}

[Supplemental material is available for this article.]

Our prior knowledge determines how we interpret and process incoming information. This preexisting knowledge is organized in associative structures called "schemas" that are based on multiple episodes, adapt in light of novel information but lack unit detail related to specific episodes (Ghosh and Gilboa 2014). It has been known for long that schemas assist text comprehension as well as memory encoding and recall (Bartlett 1932; Bransford and Johnson 1972; Anderson and Pearson 1984). The neural underpinnings of schemas and schema-related information processing, however, have been elucidated only over the past few years. Converging lines of evidence from rodent studies and human neuroimaging and patient studies point to the medial prefrontal cortex (mPFC) as a key area for detecting schema-related information and the integration of this information into the neocortical schema (van Kesteren et al. 2010b; Tse et al. 2011; Ghosh et al. 2014; Brod et al. 2015). At the same time, the hippocampus, highly relevant for the encoding of novel (i.e., schema-unrelated) information (Knight 1996; Eichenbaum 2004), is less involved in the processing of information for which a relevant schema exists (Tse et al. 2007; van Kesteren et al. 2012). Although the relevance of schemas for learning and memory is widely accepted (Wang and Morris 2010; Ghosh and Gilboa 2014), the factors that influence the processing of schema-related information and the efficient use of prior knowledge are largely unknown.

Stress has a critical impact on a broad range of cognitive functions, from social cognition to learning and memory (Joëls et al. 2006; Diamond et al. 2007; Lupien et al. 2009; Roozendaal et al. 2009; Schwabe et al. 2012a; Sandi and Haller 2015). These effects of stress are mainly mediated by hormones and neurotransmitters that are released during stressful events such as glucocorticoids (cortisol in humans) and catecholamines (Roozendaal et al. 2006;

Corresponding author: lars.schwabe@uni-hamburg.de Article is online at http://www.learnmem.org/cgi/doi/10.1101//m.046003.117.
Hermans et al. 2011; Schwabe et al. 2012a,b). Prefrontal areas, including the $\mathrm{mPFC}$, and the hippocampus are among the primary targets of these stress mediators (de Kloet et al. 2005) and stress may impair prefrontal and hippocampal functioning (Kim and Diamond 2002; Diamond et al. 2006; Arnsten 2009; Schwabe and Wolf 2009, 2012). Based on these findings it can be hypothesized that stress may interfere with the processing of schema-related information. Indeed, recent evidence indicated that stress impairs schema-related learning (Kluen et al. 2017), yet the underlying neural mechanisms remain completely unknown. We hypothesized that stress would impair information processing in the medial prefrontal cortex and the hippocampus, leading to altered schema-related processing and impaired subsequent memory.

Thus, the present experiment investigated the impact of acute stress on the neural processing of schema-related information. To this end, we exposed healthy individuals to the Trier social stress test (TSST) (Kirschbaum et al. 1993) or a control manipulation before they completed, in an MRI scanner, a task that was designed to activate preexisting knowledge structures and that allowed us to assess the encoding of schema-related information. Specifically, participants were first presented with a category word (e.g., "bathroom," Fig. 1) for which all individuals should have prior knowledge that fulfills the criteria of a schema (Ghosh and Gilboa 2014). Participants then encoded nouns for which they were asked to indicate whether the word was related (e.g., "shower") or unrelated (e.g., "kayak") to the activated schema. In addition, we presented trials in which participants were required to

(C) 2018 Vogel et al. This article is distributed exclusively by Cold Spring Harbor Laboratory Press for the first 12 months after the full-issue publication date (see http://learnmem.cshlp.org/site/misc/terms.xhtml). After 12 months, it is available under a Creative Commons License (Attribution-NonCommercial 4.0 International), as described at http://creativecommons.org/licenses/by-nc/4.0/. 
A

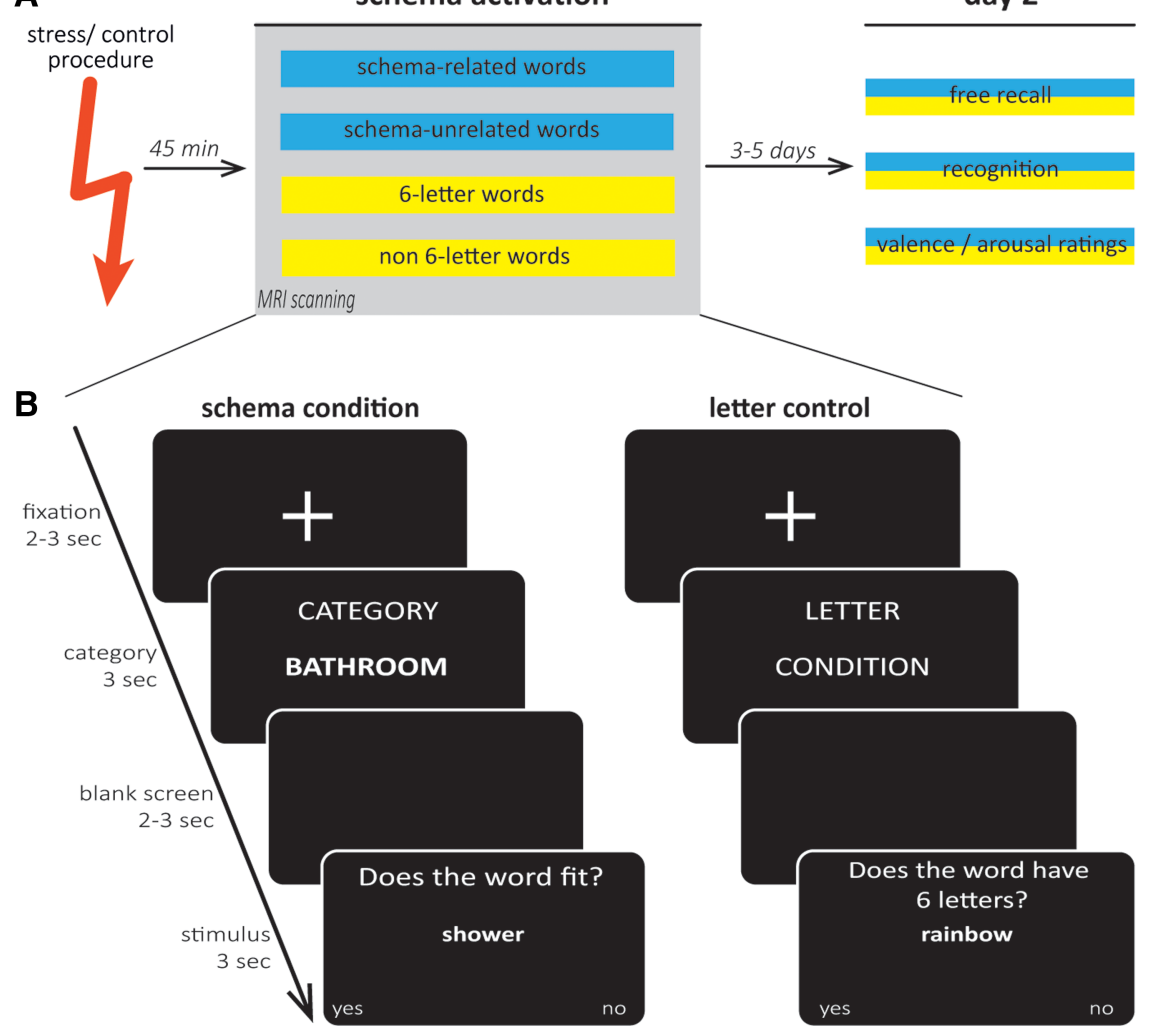

Figure 1. Experimental procedure. ( $A$ ) On day 1, participants underwent either a stress induction or a control procedure before performing the schema-related processing task in the MRI scanner. In this task, they were presented with words that either belonged to a schema or not (schema condition) or words that consisted of six letters or not (to control for the effect of mere confirmation). Four blocks of the schema condition with eight schema-related words and eight schema-unrelated words in each block were intermixed with two blocks of the letter control condition (eight words with six letters and eight words with a different length per block). Three to $5 \mathrm{~d}$ later, participants returned for a memory test (free recall and recognition) and rated all words with respect to arousal and emotional valence. (B) Trial setup and timing for the schema condition (left) and the letter control condition (right) during day 1.

indicate whether a word had six letters or not. This letter control condition served two purposes. First, it allowed us to compare the processing of schema-related information to the processing of information that belongs to a category that does not possess the features of a schema. Second, we aimed also at examining the proposed beneficial effect of a schema on memory and the impact of stress on schema-based memory as assessed during free recall and recognition tests $3-5 \mathrm{~d}$ after encoding. However, because it is known that confirming the belongingness of an item to a category already facilitates subsequent memory (Craik and Tulving 1975), a putative "schema-effect" on memory could simply be owing to the positive response to the relatedness question during encoding. The letter control condition, however, enabled us to dissociate such a confirmation effect from the impact of a schema on memory.

\section{Results}

\section{Successful stress induction prior to schema-related processing}

Exposure to the TSST resulted in a pronounced subjective and physiological stress response. As expected, participants rated the TSST as significantly more stressful, difficult, and unpleasant than the control manipulation (all $t>4, P<0.001$ ) (Table 1).
Accordingly, the TSST decreased positive mood and calmness compared with the control manipulation (time $\times$ treatment: both $F>7, P \leq 0.001$ ) (Table 1 ). In addition, the TSST resulted in increased diastolic and systolic blood pressure compared with the control manipulation (time $\times$ treatment: both $F>8$, both $P \leq$ 0.001 ) (Fig. 2), indicating successful activation of the autonomic nervous system. Finally, the TSST led to a marked increase in salivary cortisol levels (time $\times$ treatment: $\left.F_{(2.6,125.5)}=10.75, P<0.001\right)$, supporting a pronounced activation of the hypothalamus-pituitary-adrenal (HPA) axis in the stress group. As shown in Figure 2C, cortisol levels were significantly elevated before and throughout the task.

\section{Behavioral distinction between schema-related and schema-unrelated words}

As expected, participants in both groups were well able to dissociate schema-related words $(96.3 \% \pm 0.73 \%$ "related" responses) from schema-unrelated words $(10.0 \% \pm 1.24 \%$ "related" responses, main effect of relatedness: $F_{(1,48)}=$ $2386.97, P<0.001)$. The fact that participants identified almost all schema-related words as belonging to the respective schema underlines that these words represented indeed characteristic parts of the schema structure. Similarly, participants correctly classified words as six letter- vs. no six letter words in the letter control condition $(88.6 \% \pm 1.39 \%$ vs. $3.9 \% \pm 0.84 \%, \quad F_{(1,48)}=2521.12, P<$ 0.001). Importantly, stress did not affect participants' ability to correctly identify words as being related vs. unrelated to the schema category or as having six vs. more/ less than six letters (all $F<2.2$, all $P>0.15$ ).

\section{Neural networks differentiating schema-related and schema-unrelated words}

In line with previous reports (van Kesteren et al. 2010b, 2013; Tse et al. 2011; van Buuren et al. 2014; Wagner et al. 2015), the presentation of schema-related words compared with schema-unrelated words led to a pronounced activation of brain regions described to be involved in schema processing and memory encoding, i.e., the $\mathrm{mPFC}$, angular gyrus, precuneus, but also the hippocampus (all $P_{\mathrm{FWE}}<0.05$, Fig. 3, for whole-brain results, see Supplemental Table S1). Moreover, brain regions involved in language processing such as the inferior and middle temporal gyri were more activated for schema-related compared with schema-unrelated words $\left(P_{\mathrm{FWE}}\right.$ $<0.05)$. In contrast, brain regions involved in salience processing and working memory functions were more engaged for schema-unrelated words, i.e. the anterior insula, dorsal anterior cingulate cortex (ACC), and inferior frontal gyrus (all $P_{\mathrm{FWE}}<0.05$, Fig. 3; Supplemental Table S2).

Importantly, the activation of this network for schema-related words could not be explained by the mere fact that participants gave a confirmative answer as shown in the letter control trials. In 
Table 1. Subjective measures of stress on day 1 in both groups

\begin{tabular}{|c|c|c|}
\hline & Stress group & Control group \\
\hline \multicolumn{3}{|l|}{ Elevated mood } \\
\hline Before treatment & $35.16(0.77)$ & $34.60(1.04)$ \\
\hline After treatment & $29.60^{*}(1.31)$ & $34.16(1.09)$ \\
\hline End of day 1 & $32.44(1.00)$ & $33.80(0.80)$ \\
\hline \multicolumn{3}{|l|}{ Wakefulness } \\
\hline Before treatment & $30.04(0.98)$ & $31.92(1.23)$ \\
\hline After treatment & $29.88(0.94)$ & $31.44(1.11)$ \\
\hline End of day 1 & $26.08(1.06)$ & $25.68(1.16)$ \\
\hline \multicolumn{3}{|l|}{ Calmness } \\
\hline Before treatment & $33.84(0.81)$ & $32.12(1.21)$ \\
\hline After treatment & $27.52^{\star *}(1.17)$ & $32.36(1.22)$ \\
\hline End of day 1 & $32.08(0.98)$ & $33.84(0.78)$ \\
\hline \multicolumn{3}{|c|}{ Rating of stressor/control procedure } \\
\hline Difficult & $69.20^{* * *}(4.00)$ & $32.80(5.49)$ \\
\hline Unpleasant & $64.00^{* \star \star}(4.08)$ & $30.00(5.80)$ \\
\hline Stressful & $66.40^{* * *}(4.51)$ & $26.40(4.47)$ \\
\hline
\end{tabular}

Note: Data represent mean (SEM). In the mood questionnaire, higher scores represent elevated mood, wakefulness, and calmness, respectively. $\left.{ }^{* \star *}\right) P<$ $\left.\left.0.001,{ }^{* *}\right) P<0.01,{ }^{*}\right) P<0.05$ compared with the control group.

these trials, words with six letters (vs. words with more or fewer letters) did not activate the mPFC, angular gyrus, or precuneus, but engaged the occipital and temporal gyri, insula, posterior cingulate, and inferior frontal gyrus (all $P_{\mathrm{FWE}}<0.05$, Supplemental Table S3). Moreover, a category $\times$ relatedness interaction ([schema-related $>$ schema-unrelated $]>$ [six letters $>$ no six letters]) revealed that the angular gyrus, precuneus, inferior and middle temporal gyri, occipital cortex (all $P_{\mathrm{FWE}}<0.05$ ) (Supplemental Table S4), and-at trend level-the $\mathrm{mPFC}\left(P_{\mathrm{SVC}}=0.065, k=131\right.$, $T=3.73$ ) were specifically more activated for related words in the schema condition than for six letter words in the letter control condition.

\section{Stress increases hippocampal activity during schema-related processing}

In line with our hypothesis, stress had a significant impact on the neural structures involved in the processing of schema-related information. Specifically, the stress group showed stronger hippocampal activation for schema-related compared with schema-unrelated words than the control group $\left(P_{\mathrm{SVC}}=0.032, k\right.$ $=42, T=3.67$ ) (Fig. 4A). Whereas the control group showed reduced hippocampal activity when encoding schema-related (vs. schemaunrelated) words $\left(P_{\mathrm{SVC}}=0.022, k=51, T\right.$ $=3.81)$ as predicted by previous reports (van Kesteren et al. 2012), the stress group showed increased hippocampal activity for schema-related when compared with schema-unrelated words $\left(P_{\mathrm{SVC}}=0.001, k\right.$ $=196, T=4.81$ ) (Supplemental Table S1). Moreover, across groups the hippocampal activation during the presentation of schema-related compared with schemaunrelated words was associated with the cortisol response to the experimental treatment ( $r=0.338, P=0.017)$ (Fig. 4B). Although this correlation should be treated with some caution as it did not reach significance in the stress group alone, presumably due to a lack of power, our findings suggest that acute stress leads to an aberrant engagement of the hippocam- pus during the processing of schema-related information, possibly driven by heightened levels of cortisol. Notably, the enhancing effect of stress on hippocampal activity was specific to the schema condition as indicated by a significant treatment $\times$ category $\times$ relatedness interaction $\left(P_{\mathrm{SVC}}=0.046, k=20, T=3.52\right)$. Accordingly, the effect of schema-relatedness on hippocampal activity was stronger in the schema trials than in the letter control trials in the stress group $\left(P_{\mathrm{SVC}}=0.030, k=58, T=3.69\right)$ (Supplemental Table S4), whereas this was not the case in the control group (no voxel at $P$ $<0.005$, uncorrected). Moreover, we found no effect of stress on any other ROI (no voxel at $P<0.005$, uncorrected) and no effect of stress on neural activity during letter control trials, supporting that stress specifically altered hippocampal activity during schema activation.

\section{Stress leads to enhanced coupling between the hippocampus and regions involved in schema processing}

Next, we investigated functional connectivity of the hippocampal cluster that showed altered schema-related activity after stress (Fig. $5 \mathrm{~A}$ ), in order to understand if and how stress altered the crosstalk of the hippocampus with other regions in the brain. A psychophysiological interaction (PPI) analysis investigating enhanced hippocampal coupling during schema-related trials when compared with schema-unrelated trials revealed that stress enhanced schema-related hippocampal connectivity with brain areas involved in schema processing such as left inferior and superior temporal gyrus, (pre)cuneus (all $P_{\mathrm{FWE}}<0.05$ ) (Fig. 5B; Supplemental Table S5), angular gyrus $\left(P_{\mathrm{SVC}}=0.033, k=46, T=3.37\right)$, and left hippocampus $\left(P_{\mathrm{SVC}}=0.038, k=54, T=3.57\right.$; note that the seed was in the right hemisphere). In contrast, no brain region showed stronger schema-related hippocampal connectivity in the control group compared with the stress group (Supplemental Table S5). When investigating the control group separately, hippocampal connectivity during schema activation (when compared with schema-unrelated trials) was confined to the left hippocampus $\left(P_{\mathrm{SVC}}=0.046, k=11, T=3.50\right)($ Fig. $5 \mathrm{C})$, indicating that the presence of a schema did not affect hippocampal connectivity with the schema network. Within the stress group, however, we found pronounced schema-related hippocampal connectivity to the precuneus, inferior and superior left temporal gyri (all $\left.P_{\mathrm{FWE}}<0.05\right)$ (Fig. 5D), mPFC $\left(P_{\mathrm{SVC}}=0.007, k=67, T=4.23\right)$, angular gyrus $\left(P_{\mathrm{SVC}}=0.015, k=62, T=3.69 ; P_{\mathrm{SVC}}=0.044, k=102, T=3.53\right)$, and the left hippocampus $\left(P_{\mathrm{SVC}}=0.032, k=24, T=3.65\right)$, suggesting that the hippocampus was incorporated into the network
A

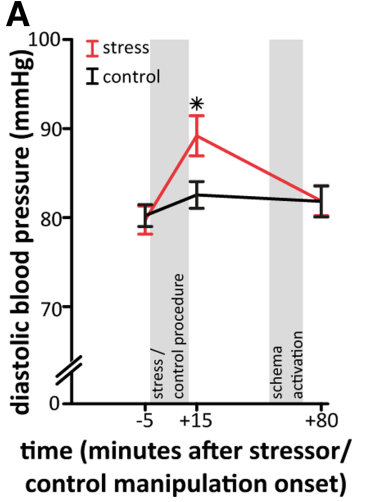

B

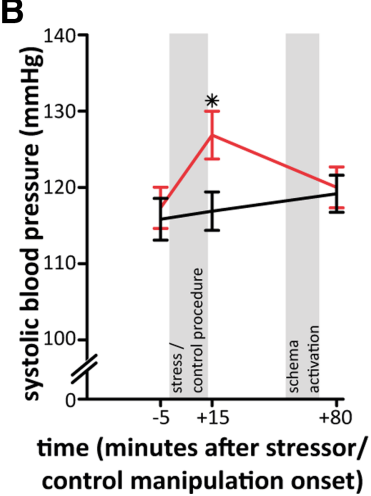

C

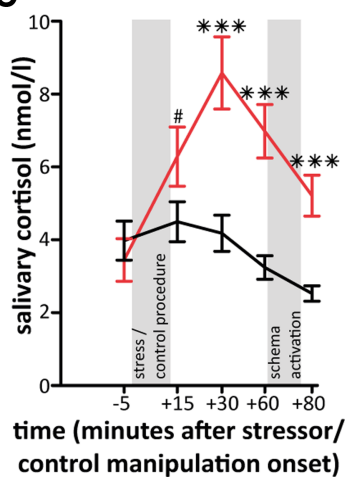

Figure 2. Physiological parameters confirmed successful stress induction. Compared with controls, participants in the stress group displayed heightened $(A)$ diastolic and $(B)$ systolic blood pressure as well as $(C)$ a pronounced increase in salivary cortisol levels after the experimental treatment. Data rep-

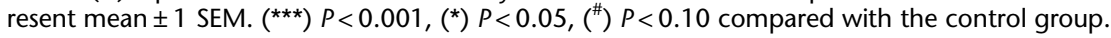



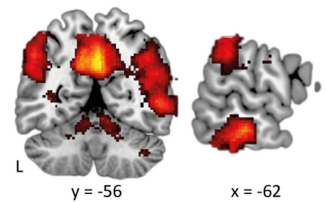

schema-related > schema-unrelated
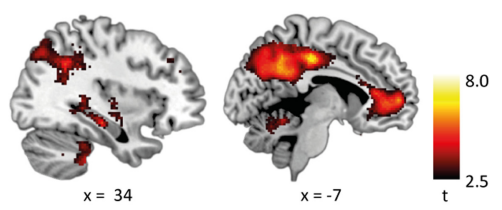

schema-unrelated > schema-related
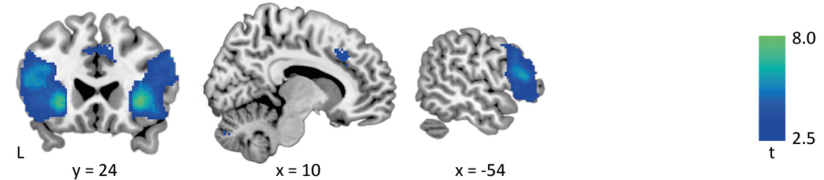

Figure 3. Brain regions differentiating schema-related and schema-unrelated words. Across groups, brain regions responding more to the presentation of schema-related words included the precuneus, angular gyrus, temporal gyrus, medial prefrontal cortex, and hippocampus. In contrast, brain regions activated in response to schema-unrelated words included the insula, dorsal anterior cingulate, and inferior frontal gyrus. Images are displayed at $P<0.005$, uncorrected, for illustrative purposes.

processing schema-related and semantic information. For the opposite PPI (schema-unrelated $>$ schema-related), the control group showed enhanced hippocampal connectivity with regions activated in response to schema-unrelated words, such as the left inferior frontal gyrus $\left(P_{\mathrm{SVC}}=0.010, k=54, T=3.83\right)$ (Supplemental Table S5), and, at trend level, the insula $\left(P_{\mathrm{SVC}}=0.055, k=43, T=3.49\right)$ however, showed no significant enhancement of hippocampal connectivity when presented with schema-unrelated words when compared with schema-related words.

To summarize, stress profoundly affected hippocampal activity and connectivity during schema activation. Participants in the control group engaged the hippocampus more when presented with schema-unrelated information as predicted by previous studies (Tse et al. 2007, 2011; van Kesteren et al. 2012), but stressed individuals activated the hippocampus more strongly in response to schema-related words. Moreover, the presence of a schema enhanced connectivity between the hippocampus and the schema network in the stress group but not the control group.

\section{Stress-induced alterations of hippocampal activity are linked to schema-based memory}

Because it is known that a schema may facilitate the encoding of schema-related information (Bartlett 1932; van Kesteren et al. 2014), we investigated in a final step how the observed stress-induced changes in hippocampal recruitment during the presentation of schema-related information were associated with subsequent memory performance. Importantly, before memory testing on day 2 the groups did not differ in any measure of stress, thus excluding a direct effect of the experimental treatment on memory recall (all $P>0.30$ ) (Table 2 ). Moreover, valence and arousal ratings did not differ between targets and lures, schema and letter and dorsal ACC $\left(P_{\mathrm{SVC}}=0.079, k=163, T=3.64\right)$. The stress group, control words, or related and unrelated words (all $P>0.10$, average valence ratings between 5.6 and 5.8 , average arousal ratings between 3.4 and 3.6).

Overall, memory performance was relatively low (Table 3), most likely due to the rather long study-test delay and the fact that the 96 words were only presented once which made the task rather difficult. Nonetheless, we observed striking schema effects on free recall and recognition performance. During free recall, participants remembered significantly more schema-related words than schema-unrelated words $\left(t_{(49)}=7.02, P<0.001\right)$. In the letter control condition, however, there was no difference in memory for six letter vs. no six letter words $\left(t_{(49)}=1.12, P=0.267\right.$, category $\times$ relatedness interaction: $\left.F_{(1,48)}=39.77, P<0.001\right)$, suggesting that the mere confirmative answer during encoding cannot explain the enhanced memory for schema-related words. Likewise, participants mentioned more schema-related but incorrect (new) words than schema-unrelated new words $\left(t_{(49)}=2.43, P=0.019\right)$. Stress, however, did not affect free recall performance (all $P>0.20$ ).

In the recognition test, participants were also better at recognizing schema-related old words than schema-unrelated old words $\left(t_{(49)}=7.80, P<0.001\right)$ and this effect was less pronounced in the letter control condition $\left(t_{(49)}=2.29, P=0.026\right.$, category $\times$ related interaction: $\left.F_{(1,48)}=4.11, P=0.048\right)$. Finally, participants committed more schema-related false alarms than schema-unrelated false alarms $\left(t_{(49)}=7.32, P<0.001\right)$, an effect that was not present in the letter control condition $\left(t_{(49)}=0.19, P=0.847\right.$; category $\times$ related interaction: $\left.F_{(1,48)}=31.36, P<0.001\right)$.

On the group level, we did not find any effects of the experimental treatment on schema-related hits (all $P>0.15$ ), possibly due to the rather low overall memory performance. In a next step, we thus focused on inter-individual differences. Across subjects the stress-induced hippocampal activation for schema-related items was associated with impaired memory performance. More precisely, although we found no association between schema-related brain activity and schema-related hits, we found a positive correlation across groups between hippocampal schema-related activity (schema-related $>$ schema-unrelated) and
A stress $>$ control

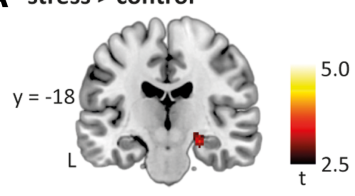

control
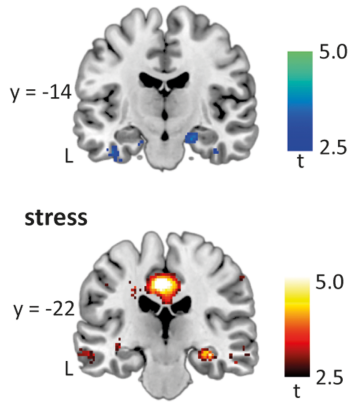

B

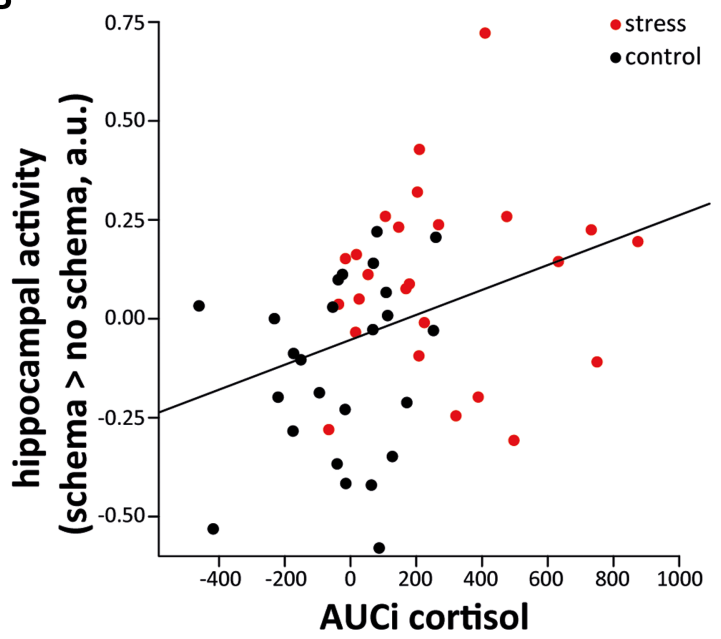

Figure 4. Stress affected hippocampal activity during schema-related processing. (A) Stress increased hippocampal activity during the presentation of schema-related when compared with schema-unrelated words. Whereas the control group engaged the hippocampus more for schema-unrelated words, the stress group showed higher hippocampal activation for schema-related words. (B) Across all participants, this schema-related hippocampal activity (cluster shown in $A$, top slice) was positively correlated with the cortisol response to the experimental treatment as reflected in the area under the curve with respect to the increase $\left(A \cup C_{i}\right)$. Images are displayed at $P<0.005$, uncorrected, for illustrative purposes. 
A

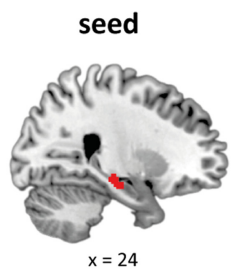

C

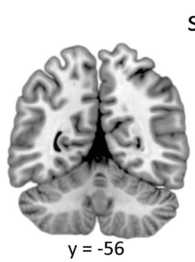

schema-related > schema-unrelated
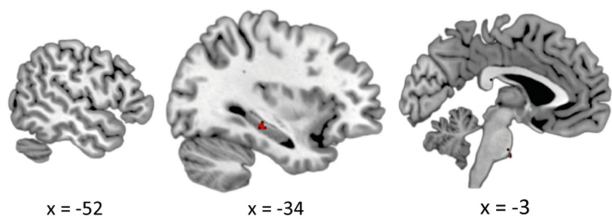

schema-related < schema-unrelated
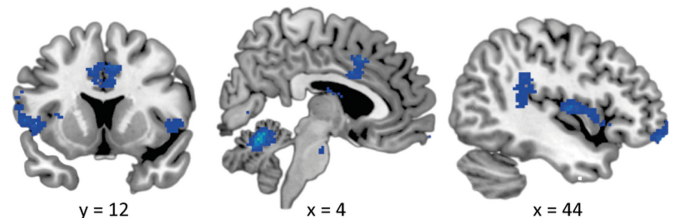

B

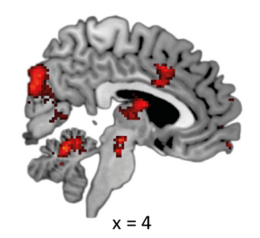

D stress $>$ control

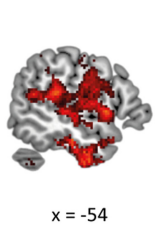

stress

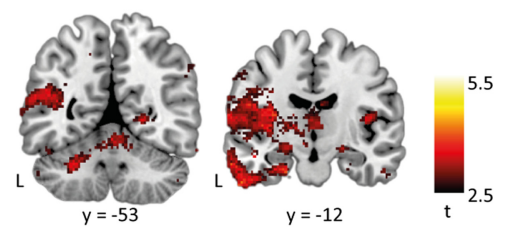

schema-related > schema-unrelated
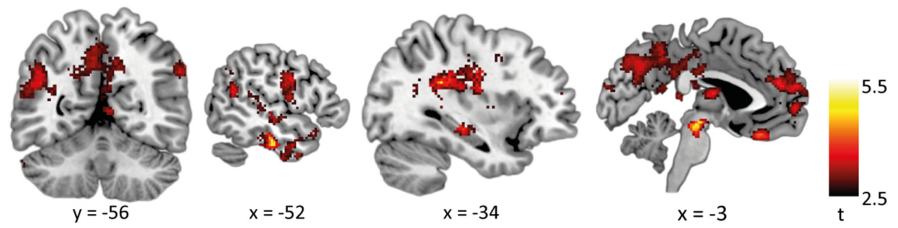

schema-related < schema-unrelated
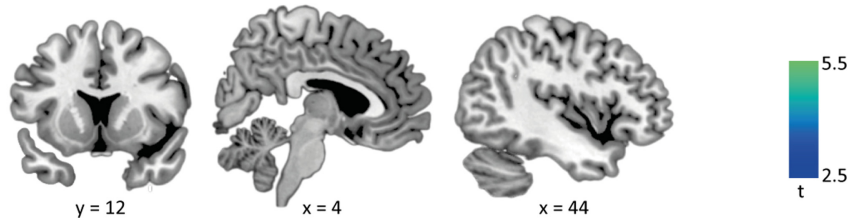

Figure 5. Stress changed hippocampal connectivity during schema-related information processing. (A) Seed region taken from the stress effect on schema-related hippocampal activity (also shown in Fig. 4A, top) for a psychophysiological interaction analysis contrasting schema-related and schema-unrelated word presentation. (B) Stress increased schema-dependent hippocampal connectivity with the cuneus (draining into the precuneus), temporal gyrus, angular gyrus, and hippocampus. (C) The control group showed schema-related hippocampal connectivity only with the left hippocampus (note that the seed was in the right hemisphere), but displayed enhanced connectivity between the hippocampus and the dorsal anterior cingulate, the insula, and the inferior frontal gyrus when presented with schema-unrelated words. (D) The stress group integrated the hippocampus into the network for schema and semantic processing (angular gyurs, precuneus, medial prefrontal cortex, and temporal gyrus) during presentation of schema-related words, but showed no significant connectivity enhancement during schema-unrelated word processing. Images are displayed at $P<0.005$, uncorrected, for illustrative purposes.

schema-related false alarms at recognition on day $2\left(P_{\mathrm{SVC}}=0.047, k\right.$ $=13, T=3.53$ [right]; $P_{\mathrm{SVC}}=0.048, k=29, T=3.51$ [left], Fig. 6). This suggests that individuals with stronger hippocampal schema-related activation were impaired in determining which schema-related words were presented on day 1 and which ones were schema-related but not shown. The correlation was specific to schema-related false alarms as the mean activity in these hippocampal clusters was not associated with schema-unrelated false alarms $(r=0.212, P=0.139$ [left] and $r=0.101, P=0.483$ [right]). However, we found no significant correlation between cortisol (area under the curve) and schema-related false alarms ( $r=$ $-0.176, P=0.222$ ).

Next, we investigated whether the stress-induced changes in hippocampal connectivity were also related to memory performance. Indeed, we found a positive association between schema-related hippocampal connectivity with the left inferior temporal gyrus and more schema-related false alarms at recognition $\left(P_{\mathrm{SVC}}=0.022, k=21, T=3.59\right)$ (Fig. 6), suggesting that a closer interaction between the hippocampus and regions involved in semantic processing leads to increased schema-related false alarm rates. Moreover, we found a negative association between hippocampus-mPFC connectivity and the number of schema-related false alarms at recognition $\left(P_{\mathrm{FWE}}<0.05\right)$ (Fig. 6), suggesting that enhanced coupling between the hippocampus and the mPFC aids the suppression of false alarms at later recognition.

Finally, we explored whether gender affected the effect of stress on memory performance. Whereas free recall was unaffected by gender (all $P>0.10$ ), we found gender differences in stress effects on hits during the recognition test such that performance was affected by stress in females only (gender $\times$ treatment $\times$ category $\times$ relatedness interaction, $\left.F_{(1,46)}=11.533, P=0.001\right)$. However, considering that our sample size was rather small to investigate gender differences, this finding should be interpreted with some caution. Details on the exploratory analysis of gender differences are reported in the Supplemental Material.

\section{Discussion}

Prior knowledge, represented as mental schema, allows us to form expectations and hence makes the world around us more predictable. Schemas further help us to organize, interpret, and remember information, thus making efficient schema-related processing essential for education. In a previous study, we demonstrated that

Table 2. Subjective and physiological measures of stress on day 2 in both groups

\begin{tabular}{lll}
\hline & Stress group & Control group \\
\hline Elevated mood & $35.28(0.52)$ & $34.88(0.94)$ \\
Wakefulness & $29.96(1.29)$ & $31.60(1.03)$ \\
Calmness & $33.80(0.75)$ & $33.24(0.97)$ \\
Systolic blood pressure $(\mathrm{mmHg})$ & $120.14(4.04)$ & $116.10(2.72)$ \\
Diastolic blood pressure $(\mathrm{mmHg})$ & $78.70(1.99)$ & $80.78(1.63)$ \\
Salivary cortisol (nmol/L) & $4.94(0.94)$ & $4.56(0.68)$ \\
\hline
\end{tabular}

Note: Data represent mean (SEM). In the mood questionnaire, higher scores represent elevated mood, wakefulness, and calmness, respectively. 
Table 3. Memory performance on day 2

\begin{tabular}{|c|c|c|}
\hline & $\begin{array}{l}\text { Stress } \\
\text { group }\end{array}$ & $\begin{array}{l}\text { Control } \\
\text { group }\end{array}$ \\
\hline \multicolumn{3}{|l|}{ Correct free recall } \\
\hline Schema-related (\%) & $12.0(2.3)^{\star \star *}$ & $9.3(1.6)^{\star \star *}$ \\
\hline Schema-unrelated (\%) & $3.4(0.9)$ & $1.6(0.5)$ \\
\hline Letter control: 6-letter words (\%) & $2.3(1.4)$ & $1.3(0.5)$ \\
\hline Letter control: no 6-letter words (\%) & $1.0(0.5)$ & $0.8(0.5)$ \\
\hline \multicolumn{3}{|l|}{ Incorrect free recall } \\
\hline Schema-related $(N)$ & $2.8(0.6)$ & $3.0(0.5)^{\star}$ \\
\hline Schema-unrelated $(N)$ & $2.2(0.5)$ & $1.8(0.4)$ \\
\hline \multicolumn{3}{|l|}{ Recognition: hits } \\
\hline Schema-related (\%) & $48.8(4.7)^{\star \star \star}$ & $51.9(3.5)^{\star \star \star}$ \\
\hline Schema-unrelated (\%) & $34.0(3.7)$ & $35.6(3.7)$ \\
\hline Letter control: six-letter words (\%) & $35.3(4.8)$ & $39.8(4.6)^{*}$ \\
\hline $\begin{array}{l}\text { Letter control: no six-letter words } \\
\text { (\%) }\end{array}$ & $30.3(3.9)$ & $30.0(4.0)$ \\
\hline \multicolumn{3}{|l|}{ Recognition: false alarms } \\
\hline Schema-related (\%) & $49.8(4.0)^{\star \star \star}$ & $53.1(3.9)^{\star \star \star}$ \\
\hline Schema-unrelated (\%) & $31.0(2.6)^{\circ}$ & $40.5(3.5)$ \\
\hline Letter control: six-letter words (\%) & $36.0(3.5)$ & $34.5(3.7)$ \\
\hline $\begin{array}{l}\text { Letter control: no six-letter words } \\
\text { (\%) }\end{array}$ & $29.8(3.5)^{\#}$ & $39.8(3.8)$ \\
\hline
\end{tabular}

Note: Data represent mean (SEM). $\left.\left.{ }^{* * *}\right) P<0.001,{ }^{* *}\right) P<0.01,\left({ }^{*}\right) P<0.05$ compared with the unrelated condition (schema-unrelated or no-6-letter words, respectively). ( $\left.{ }^{\circ}\right) P<0.05$, (") $P<0.10$ compared with the control group.

stress impairs schema-related learning (Kluen et al. 2017). Here, we examined the impact of acute stress on the underlying neural mechanism. Stress increased the recruitment of the hippocampus in schema-related processing and led to an aberrant involvement of the hippocampus in the schema network during schema activation. Although there was no stress effect on memory at the group level, which might be due to a lack of statistical power, these stress-induced alterations in schema-related processing were associated with increased false alarms during recognition.

We used a schema concept that was leaned on the classical cognitive psychological schema literature (Bartlett 1932; Rumelhart 1980; Brewer and Treyens 1981). This schema concept and the way it was operationalized differs from schema conceptualizations used in rodents and other neuroimaging studies (Tse et al. 2007; van Kesteren et al. 2010a, b; Tse et al. 2011; Brod et al. 2015). In the current study, we compared multiple words that were all related to a certain category with words that were not related to that category or to the other words presented. Nonetheless, the neural structures activated during the presentation of schema-relevant words were similar to what has been reported in these previous studies, suggesting that the processing of schema-related information involves a specific network of areas, irrespective of the very specific features of the activated schema. In line with previous studies (van Kesteren et al. 2010a, b, 2012; Tse et al. 2011; Brod et al. 2015), we observed activation of the MPFC, precuneus, and angular gyrus during the processing of schema-related information. Notably, by means of our letter control condition we could demonstrate that this network is not activated when presented stimuli merely belong to any arbitrary category but that it appears to be specifically recruited when information is processed for which an associative knowledge structure exists that was built over multiple episodes (i.e., a schema). Likewise, information that is unrelated to a schema activated other brain regions, such as the dorsal ACC, the anterior insula, and the inferior frontal gyrus, that are involved in salience, conflict processing, or general working memory (Courtney et al. 1997; Botvinick et al. 1999; Menon and Uddin 2010).

Most important, the brain areas involved in schema-related processing were affected by stress. Participants in the control con- dition showed increased activity of the schema network (mPFC, precuneus, angular gyrus) but not the hippocampus when processing schema-related information. Furthermore, hippocampal connectivity with this network was unaffected by the presence of a schema in controls. This pattern is in line with a recent model of schema-related processing that postulates that if a relevant schema exists, information processing is mainly guided by the mPFC communicating with neocortical areas representing the schema, whereas the hippocampus and its detailed episodic processing is less involved (van Kesteren et al. 2012). When stressed, however, the hippocampus was activated by schema-related information and incorporated into the schema network during schema-related trials. The involvement of an area specialized for detailed episodic processing of novel information (Knight 1996; Eichenbaum 2004) may likely interfere with the more abstract, knowledge-based schema-related processing and indeed our data show that the activity of the hippocampus in schema-related processing was associated with impaired memory (see below). Moreover, the activity of the hippocampus in schema-related processing was correlated with cortisol levels across all participants (but not in the stress group alone), suggesting that this major stress hormone might play a role in the aberrant engagement of the hippocampus in schemarelated processing. Interestingly, a very recent behavioral study demonstrated that both stress and the administration of hydrocortisone resulted in a learning impairment for schema-related items (Kluen et al. 2017), which is in line with our current finding. Furthermore, our results concur with previous reports that identified glucocorticoids as a major modulator of prefrontal and hippocampal functioning (de Quervain et al. 2003; Roozendaal et al. 2004; Wiegert et al. 2006; Henckens et al. 2011, 2012; Schwabe et al. 2012b). Cortisol exerts its actions in the brain through mineralocorticoid and glucocorticoid receptors (MR and GR, respectively) (de Kloet et al. 1986). Based on accumulating evidence that membrane-bound MR mediate rapid, nongenomic glucocorticoid actions (Joëls et al. 2012) and that that these receptors are implicated in how we approach a situation and to what extent we utilize available information (Oitzl and de Kloet 1992; Schwabe et al. 2010; Vogel et al. 2016), it is tempting to speculate that membrane-bound MR were also involved in stress and cortisol effects on neural schema-related processing. The correlation with cortisol, however, should not be taken as evidence that cortisol acted in isolation. For instance, there is compelling evidence that glucocorticoids act synergistically (or antagonistically) with catecholamines to affect cognition and its neural underpinnings (Roozendaal et al. 2004, 2006, 2009; Schwabe et al. 2012b; Hermans et al. 2014).

Although this study focused mainly on schema-related processing and its modulation by stress, we also found better free recall and recognition performance for items that were schema-related compared with schema-unrelated items, which corroborates previous reports of schema-based memory (Bartlett 1932; van Kesteren et al. 2010b). Using the letter control condition, we could show that this memory enhancement for schema-related items was indeed due to the existing schema and not just to the relation to any arbitrary category or the mere positive response to the relatedness question. The schema-effect on memory, however, was not only reflected in superior memory for the schema-related items that were presented during encoding but also in more incorrect memories of or false alarms to items that were related to the schemas activated during encoding, providing further evidence that a schema may also bias remembering (Bartlett 1932; Brewer and Treyens 1981; Roediger and McDermott 1995). Our fMRI data revealed that this schema-related enhancement of false alarms was negatively correlated with hippocampal-mPFC crosstalk. In other words, the efficient use of an existing schema, leading to specific memories, appears to require communication between the mPFC 
A

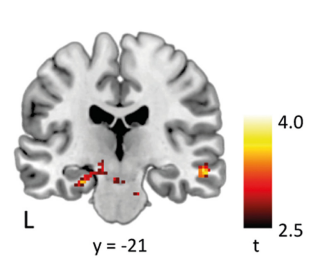

B

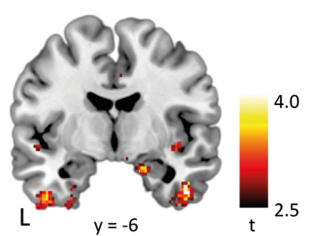

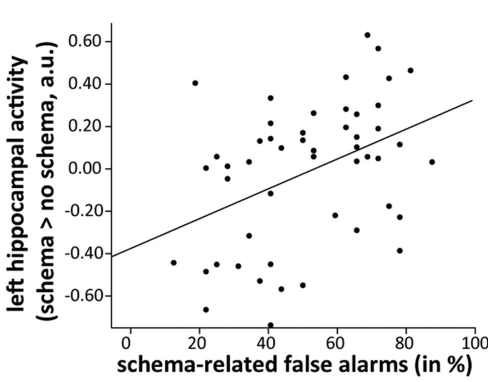

C
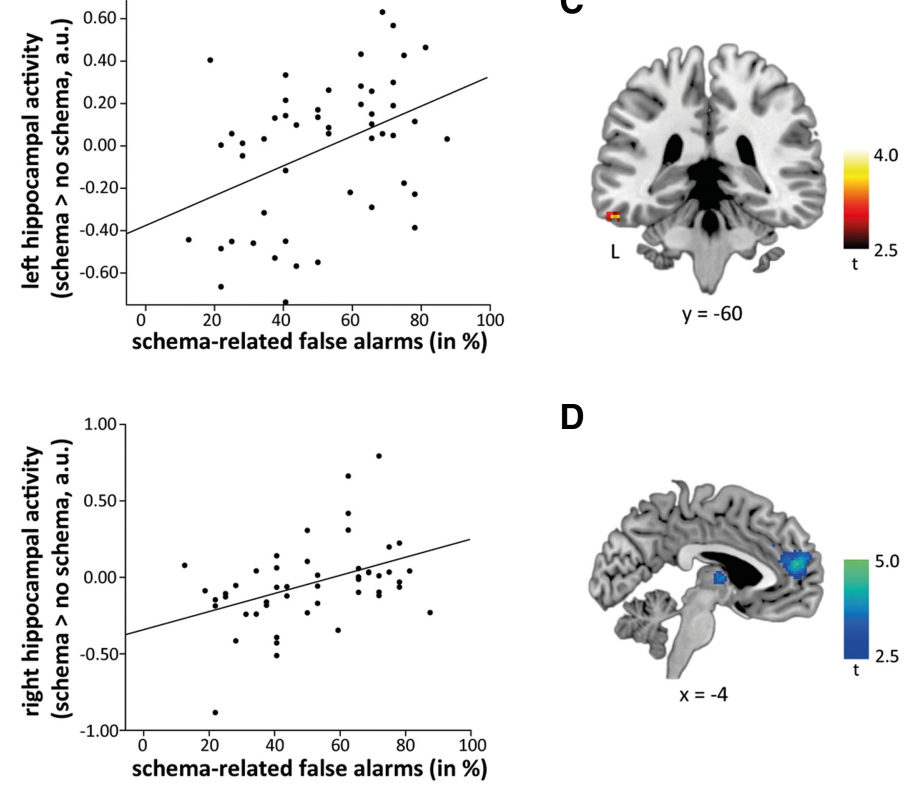

D

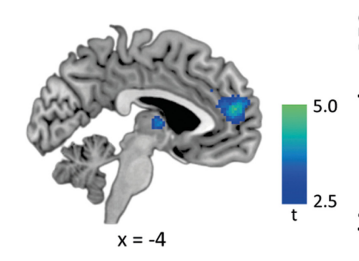

Figure 6. Hippocampal activity and connectivity during schema-related information processing predicted false alarm rates. ( $A$, left and $B$, right) hippocampal activity in response to schema-related words was associated across groups with increased schema-related false alarms on day 2 . ( $C$ ) Schema-related hippocampal connectivity to the semantic network enhanced schema-related false alarms at recognition. Please note that removing the participant with the highest connectivity estimates did not change the result. $(D)$ In contrast, schema-related hippocampal connectivity to the medial prefrontal cortex (mPFC) was associated with fewer schema-related false alarms. Images are displayed at $P<0.005$, uncorrected, for illustrative purposes.

and hippocampus. Despite the fact that PPI data provide no insights into the direction of communication, this finding is generally in line with the view that the mPFC, as the schema-relevance detector, needs to communicate with the hippocampus to allow efficient use of the schema (van Kesteren et al. 2012). Hippocampal crosstalk with inferior temporal cortices implicated in semantic processing and visual memory (Miller et al. 1991; Vandenberghe et al. 1996), however, was associated with a reduced specificity of the memory for schema-related information that was actually presented. Considering that the MPFC and the inferior temporal gyrus are both considered to be part of the same network, the opposing relationships between their connectivity with the hippocampus and schema-related false alarm rates are somewhat surprising. Future studies focusing on the specific roles of each of the nodes of the network activated by schema-related information may shed more light on the precise mechanisms underlying these associations.

Whereas the current project focused primarily on the effects of stress on the (neural) processing of schema-related and schema-unrelated information, another interesting question would be if and how stress affects the initial acquisition of a schema. While this topic was beyond the scope of our project, previous studies on stress-induced changes in learning and memory suggest that stress effects on schema acquisition would likely depend on different factors such as the valence of the schema (Schwabe et al. 2008; Zoladz et al. 2011) or the timing of the acquisition relative to the stressful encounter (Joëls et al. 2006; Zoladz et al. 2011; Schwabe et al. 2012a; Vogel and Schwabe 2016). According to these reports, stress is expected to enhance learning when the task rapidly follows the stressful encounter and even more so for emotional learning material. In contrast, schema learning should be impaired if the learning task is delayed by more than $75 \mathrm{~min}$.

To conclude, we show here that stress alters hippocampal activity and connectivity when participants process schema-related information and that these changes may translate into impaired schema-based learning. These findings converge with recent evidence suggesting that stress makes memories more rigid, hampering the flexible use of previously acquired or currently available information (Dandolo and Schwabe 2016; Vogel et al. 2016). Our findings may have important implications for educational contexts in which students frequently encounter stressful events (Vanelst et al. 2012) and learning of information that is congruent with prior knowledge is crucial. Moreover, our findings may aid our understanding of stress-related mental disorders in which information processing against the background of preexisting knowledge is impaired (Beck and Clark 1988; Moritz and Woodward 2005).

\section{Materials and Methods}

\section{Participants and experimental design}

Fifty healthy, right-handed volunteers (25 men, 25 women) participated in this experiment. All participants had normal or corrected-to-normal vision (mean age \pm SEM: $25 \pm 0.5 \mathrm{yr}$ ) and were screened for the following exclusion criteria: current nonadmissibility to the MRI scanner, current medication intake, lifetime history of any neurological or psychiatric disorders, regular smoking, and use of hormonal contraceptives. The study protocol was approved by the review board of the German Psychological Society (LS 062014_B). All participants provided written informed consent and received a moderate monetary compensation for study participation.

In a mixed design with the within-subjects factor schemarelatedness (related vs. unrelated words) and the between-subjects factor treatment (stress vs. control manipulation), participants were randomly assigned to the stress (13 females, 12 males) or control condition (12 females, 13 males) while balancing for gender. No participant was excluded prior to analyses.

\section{Experimental procedure and behavioral task}

Participants were tested on two experimental days, with an interval of 3-5 d (mean delay $3.7 \mathrm{~d}$, median $3 \mathrm{~d}$ ). All testing took place between 1 and 8 p.m. 


\section{Day 1}

Upon their arrival at the MRI scanning facility of the University Medical Center Hamburg-Eppendorf, participants provided a saliva sample for subsequent cortisol analysis (see below), their vital signs (blood pressure, heart rate) were measured using a blood pressure monitor with arm cuff (Omron Healthcare), and they completed a German questionnaire assessing subjective mood on three scales: depressed vs. elevated mood, sleepiness vs. wakefulness, and restlessness vs. calmness (MDBF) (Steyer et al. 1994). After these baseline measurements, participants underwent either the TSST (TSST) (Kirschbaum et al. 1993) or a nonstressful control procedure. The TSST is a standardized 15-min protocol to induce stress in laboratory settings in humans. It resembles a job interview consisting of a preparation phase, a public speech about the participant's eligibility for a job tailored to their interests, and a difficult mental arithmetic task (counting backward from 2043 in steps of 17). Throughout the TSST, participants were videotaped and evaluated by two strict, nonreinforcing committee members. In contrast, the control procedure entailed a private speech about a topic of the participant's choice followed by a simple mental arithmetic task (counting forward from 0 in steps of 15), without being videotaped or evaluated by a committee. Afterwards, participants' vital signs were measured again, they provided another saliva sample, completed the MDBF again, and rated the difficulty, unpleasantness, and stressfulness of the experimental treatment on a scale from 0 ("not at all") to 100 ("very much"). Participants were then prepared for scanning and gave another saliva sample. In the scanner, they first performed an unrelated task that is beyond the scope of the present manuscript and will be described elsewhere. About $45 \mathrm{~min}$ after the stressor/control manipulation, when cortisol levels were expected to have reached their peak, participants gave another saliva sample and the schema activation task started. In this task (Fig. 1), participants were first presented with the name of a category (e.g., "bathroom") for which most likely every individual has prior knowledge that fulfills the criteria of a schema (Ghosh and Gilboa 2014). The category name was followed by $16 \mathrm{com}$ mon nouns which were either category-related (e.g., "shower," eight words per category) or not (e.g., "kayak," eight unrelated words; word length was equated between category-related and category-unrelated words, word order was randomized). Each word was presented for $3 \mathrm{sec}$ and participants were instructed to memorize the word and to indicate by pressing one of two buttons whether the word was related to the presented category or not; the assignment of buttons to answer options was randomized over trials. Between words, there was a jittered interval of $2-3$ sec with the random jitter uniformly drawn from this range. Between categories, a jittered fixation cross $(2-3 \mathrm{sec})$ was presented. In total, four categories were shown (bathroom, garden, office, restaurant), each followed by eight related and eight unrelated words, resulting in 32 schema-related and 32 schema-unrelated words. The assignment of words as schema-related and -unrelated was based on a pilot study $(n=12)$, in which we also confirmed that the words of the different categories as well as the words used in the letter control condition (see below) were comparable in terms of emotionality. Because it is known that a confirmative answer results in better memory for the respective stimulus, irrespective of any schema-effects (Craik and Tulving 1975), we additionally included two blocks with a confirmation control. In these blocks, participants saw the cue "letter condition" followed by 16 words, should also try to memorize the words, and decide whether the words had six letters or not. The timing for these "letter blocks" was identical to the timing for the schema blocks. Block order was random except that the two letter control blocks were separated by at least one schema block. Finally, an anatomical scan was obtained and participants could leave the scanning facility after a last saliva sample, vital signs, and mood measurement.

\section{Day 2}

Three to $5 \mathrm{~d}$ after day 1, participants came to the laboratory and again provided a saliva sample, vital signs measurement, and com- pleted the MDBF. Next, they were asked to freely recall all the words they had seen on day 1 . In a subsequent recognition test, participants were presented with all 96 old words that were presented on day 1 and 96 new lures ( 32 related and 32 unrelated to the schemas presented on day 1, 16 words with 6 letters, and 16 words with more or fewer than 6 letters) and asked to decide whether a word was old (presented on day 1) or new. Each word was displayed individually for a maximum of $3 \mathrm{sec}$ on a computer screen. Word order was randomized. If the participants answered "old," they were further asked to indicate their confidence on a scale from 1 ("not sure at all") to 4 ("very sure"). As the number of targets and lures differed between the schema condition and the letter control condition, all hits and false alarm rates were then transformed to percentages.

The assignment of words to targets (i.e., encoding on day 1 ) or lures (presented as new words during recognition testing) was counterbalanced across participants. Finally, participants were asked to rate the subjective valence and arousal of all words (targets and lures) on two scales ranging from 1 ("very negative"/"not at all arousing") to 9 ("very positive"/"very arousing").

\section{Saliva sampling and analysis}

Participants collected six saliva samples using Salivette (Sarstedt) collection devices. The samples were stored at $-18^{\circ} \mathrm{C}\left(-0.4^{\circ} \mathrm{F}\right)$ at the end of each test day and thawed for analyses at the end of data acquisition. From saliva, we analyzed the fraction of free cortisol using a commercially available chemiluminescence immunoassay (IBL, Tecan) with a lower detection limit of 0.33 $\mathrm{nmol} / \mathrm{L}$. All intra- and inter-assay coefficients of variance were below $10 \%$.

\section{Statistical analysis of physiological and behavioral data}

Data on mood, vital signs, and salivary cortisol were analyzed using mixed-design analyses of variance (ANOVA) with the betweensubjects factor treatment (TSST vs. control manipulation) and the within-subjects factor time after stress/control manipulation onset to test whether the stress induction was successful. $T$-tests were used to investigate post hoc group differences, to analyze group differences in the ratings of the stress/control manipulation, and to test for differences in stress measures on day 2. Performance on day 1 (judging whether words were schema-related or not, or had six letters or not, respectively) was averaged over trials and subjected to mixed-design ANOVAs with the between-subjects factor treatment and the within-subjects factors relatedness (related vs. unrelated) and category (schema vs. letter control). Similar ANOVAs were implemented to analyze correctly mentioned words at free recall, hits, and false alarms during recognition. Incorrectly mentioned words during free recall were classified as schema-related words (belonging to one of the conceptual schemas presented on day 1) or schema-unrelated words and analyzed using ANOVAs with the between-subjects factor treatment and the within-subjects factor relatedness. Valence and arousal ratings were analyzed using mixed-design ANOVAs with the betweensubjects factor treatment and the within-subjects factors relatedness, category, and memory (old vs. new). All analyses were performed in SPSS Statistics 22 (IBM), the $\alpha$-level was set to 0.05 (two-tailed), and Greenhouse-Geisser correction was applied when necessary.

\section{MRI data acquisition and analysis}

During task performance, MRI measurements were obtained using a 3T Skyra scanner (Siemens) equipped with a 32-channel coil. A sequence sensitive to the blood-oxygenation level dependent (BOLD) response was used to measure brain activity during schema activation with the following parameters: 27 transversal slices, slice thickness $=3 \mathrm{~mm}$, distance factor $20 \%$, repetition time $(\mathrm{TR})=2.00$ sec, echo time $(\mathrm{TE})=30 \mathrm{msec}$, effective voxel size $=3.0 \times 3.0 \times 3.0$ $\mathrm{mm}$. We also acquired magnetic (B0) field maps to unwarp the functional images and a high-resolution T1-weighted anatomical 
image $(\mathrm{TR}=2.5 \mathrm{sec}, \mathrm{TE}=2.12 \mathrm{msec}, 256$ slices, voxel size $=0.8 \times$ $0.8 \times 0.9 \mathrm{~mm})$.

All fMRI data were visually inspected for artifacts and then preprocessed and analyzed in SPM12 (Wellcome Trust Centre for Neuroimaging) using general linear modeling (GLM). The first three functional images were discarded to allow for T1 equilibration. Remaining functional images were spatially realigned and unwarped, coregistered to the structural image, normalized to MNI space, and spatially smoothed using the default $8 \mathrm{~mm}$ FWHM Gaussian kernel. No participant moved $3 \mathrm{~mm}$ (voxel size) or more. To assess neural activity related to schema activation and the effects of treatment, we used a model including separate 3-sec-boxcar regressors for the presentation of schema-related and schema-unrelated words, as well as six-letter words and no-six-letter words in the letter control blocks. Moreover, we included 3-sec-boxcar regressors for the presentation of the words describing the category/letter control condition and spikes for button presses. All regressors were convolved with the canonical hemodynamic response function and six realignment parameters were added to account for residual motion. Full-factorial designs were used to test for activation differences depending on schema-relatedness, category, and treatment. Behavioral covariates were added to the second-level GLMs where indicated to assess the relationship between neural activation and performance. Moreover, to correlate brain activity with the cortisol response to treatment, we calculated the area under the curve with respect to the increase during day 1 (AUCi) (Pruessner et al. 2003) and extracted parameter estimates from the functional region of interest showing stress-induced activity enhancement for schema-related words (right hippocampus, see Results and Fig. 4A), averaging over all significant voxels at $P<0.005$, uncorrected, using MarsBaR. To investigate the effects of stress on hippocampal connectivity during schema-related processing, we used the "Psycho-Physiological Interaction" tool as implemented in SPM12 assessing connectivity which was stronger for schema-related than schema-unrelated words. The seed region was the same right hippocampal cluster showing stress-induced activity enhancement for schema-related words. Again, a full-factorial design was used to test for group differences. For all whole-brain analyses, we used a cluster-defining threshold of $P<0.001$ and a cluster-probability of $P<0.05$ familywise error (FWE) corrected for multiple comparisons (complete whole-brain results are provided in the Supplemental Material). For our regions of interest (ROIs, hippocampus, angular gyrus, precuneus, mPFC, left inferior frontal gyrus, left inferior temporal gyrus, and insula), we implemented small volume correction (SVC) using an initial threshold of $P<0.005$, uncorrected to enhance sensitivity, followed by voxel-wise FWE-correction $(P<0.05)$ for multiple comparisons within ROIs. The results obtained by SVC are indicated by " $P_{\mathrm{SVC}}$," all other results are based on whole-brain analyses. We used only anatomical masks for SVC, taken from the Harvard-Oxford atlas using a probability threshold of 50\%. For the mPFC, we used the masks for frontal medial cortex and ACC. All images are displayed at $P<0.005$, uncorrected, for illustrative purposes.

Finally, we tested whether there were differences in scanto-scan motion between groups that may have affected our connectivity analyses. We thus calculated framewise displacement (FD) using the BRAMILA tools (Power et al. 2012). Importantly, average FD was overall rather small and did not differ between groups (control group mean $\mathrm{FD} \pm \mathrm{SD}$ : $0.19 \pm 0.6 \mathrm{~mm}$; stress group: $0.20 \pm$ $0.11 \mathrm{~mm}, P=0.533)$. Potential group differences in functional connectivity are therefore unlikely to be driven by differences in motion.

\section{Acknowledgments}

We gratefully acknowledge the technical assistance of Lukas Mönch, Daniel Kutzner, and Wiebke Schmidt. Furthermore, we thank Lisa Abels, Mewes Muhs, Rosa Maria Rühling, Amina Shah, and Sonja Timmermann for their help during data collection. This project was supported by the German Research Foundation (DFG, SCHW1357/14-1). The authors declare no competing financial interests.

\section{References}

Anderson RC, Pearson PD. 1984. A schema-theoretic view of basic processes in reading comprehension. In Handbook of reading research (ed. PD Pearson, R Barr, ML Kamil). Lawrence Erlbaum, Mahwah, NJ.

Arnsten AFT. 2009. Stress signalling pathways that impair prefrontal cortex structure and function. Nat Rev Neurosci 10: 410-422.

Bartlett FC. 1932. Remembering: a study in experimental and social psychology. Cambridge University Press, Cambridge.

Beck AT, Clark DA. 1988. Anxiety and depression: an information processing perspective. Anxiety Res 1: 23-36.

Botvinick M, Nystrom LE, Fissel K, Carter CS, Cohen JD. 1999. Conflict monitoring versus selection-for-action in anterior cingulate cortex. Nature 402: 179-181.

Bransford JD, Johnson MK. 1972. Contextual prerequisites for understanding-some investigations of comprehension and recall. I Verb Learn Verb Behav 11: 717-726.

Brewer WF, Treyens JC. 1981. Role of schemata in memory for places. Cogn Psychol 13: 207-230.

Brod G, Lindenberger U, Werkle-Bergner M, Shing YL. 2015. Differences in the neural signature of remembering schema-congruent and schema-incongruent events. Neuroimage 117: 358-366.

Courtney SM, Ungerleider LG, Keil K, Haxby JV. 1997. Transient and sustained activity in a distributed neural system for human working memory. Nature 386: 608-611.

Craik FM, Tulving E. 1975. Depth of processing and the retention of words in episodic memory. J Exp Psychol Gen 104: 268-294.

Dandolo LC, Schwabe L. 2016. Stress-induced cortisol hampers memory generalization. Learn Mem 23: 679-683.

de Kloet ER, Reul JM, de Ronde FS, Bloemers M, Ratka A. 1986. Function and plasticity of brain corticosteroid receptor systems: action of neuropeptides. I Steroid Biochem 25: 723-731.

de Kloet ER, Joels M, Holsboer F. 2005. Stress and the brain: from adaptation to disease. Nat Rev Neurosci 6: 463-475.

de Quervain DJ, Henke K, Aerni A, Treyer V, McGaugh JL, Berthold T, Nitsch RM, Buck A, Roozendaal B, Hock C. 2003.

Glucocorticoid-induced impairment of declarative memory retrieval is associated with reduced blood flow in the medial temporal lobe. Eur $J$ Neurosci 17: 1296-1302.

Diamond DM, Campbell AM, Park CR, Woodson JC, Conrad CD, Bachstetter AD, Mervis RF. 2006. Influence of predator stress on the consolidation versus retrieval of long-term spatial memory and hippocampal spinogenesis. Hippocampus 16: 571-576.

Diamond DM, Campbell AM, Park CR, Halonen J, Zoladz PR. 2007. The temporal dynamics model of emotional memory processing: a synthesis on the neurobiological basis of stress-induced amnesia, flashbulb and traumatic memories, and the Yerkes-Dodson law. Neural Plast 2007: 60803.

Eichenbaum H. 2004. Hippocampus: cognitive processes and neural representations that underlie declarative memory. Neuron 44: 109-120.

Ghosh VE, Gilboa A. 2014. What is a memory schema? A historical perspective on current neuroscience literature. Neuropsychologia 53: 104-114.

Ghosh VE, Moscovitch M, Melo Colella B, Gilboa A. 2014. Schema representation in patients with ventromedial PFC lesions. J Neurosci 34: 12057-12070.

Henckens MJAG, van Wingen GA, Joels M, Fernandez G. 2011. Time-dependent corticosteroid modulation of prefrontal working memory processing. Proc Natl Acad Sci 108: 5801-5806.

Henckens MJAG, Pu Z, Hermans EJ, Van Wingen GA, Joels M, Fernandez G. 2012. Dynamically changing effects of corticosteroids on human hippocampal and prefrontal processing. Hum Brain Mapp 33: 2885-2897.

Hermans EJ, van Marle HJF, Ossewaarde L, Henckens MJAG, Qin S, van Kesteren MTR, Schoots VC, Cousijn H, Rijpkema M, Oostenveld R, et al. 2011. Stress-related noradrenergic activity prompts large-scale neural network reconfiguration. Science 334: 1151-1153.

Hermans EJ, Henckens MJ, Joels M, Fernandez G. 2014. Dynamic adaptation of large-scale brain networks in response to acute stressors. Trends Neurosci 37: 304-314.

Joëls M, Pu ZW, Wiegert O, Oitzl MS, Krugers HJ. 2006. Learning under stress: how does it work? Trends Cogn Sci 10: 152-158.

Joëls M, Sarabdjitsingh RA, Karst H. 2012. Unraveling the time domains of corticosteroid hormone influences on brain activity: rapid, slow, and chronic modes. Pharmacol Rev 64: 901-938.

Kim JJ, Diamond DM. 2002. The stressed hippocampus, synaptic plasticity and lost memories. Nat Rev Neurosci 3: 453-462.

Kirschbaum C, Pirke KM, Hellhammer DH. 1993. The 'trier social stress test' - a tool for investigating psychobiological stress responses in a laboratory setting. Neuropsychobiology 28: 76-81. 
Kluen LM, Nixon P, Agorastos A, Wiedemann K, Schwabe L. 2017. Impact of stress and glucocorticoids on schema-based learning. Neuropsychopharmacology 42: 1254-1261.

Knight RT. 1996. Contribution of human hippocampal region to novelty detection. Nature 383: 256-259.

Lupien SJ, McEwen BS, Gunnar MR, Heim C. 2009. Effects of stress throughout the lifespan on the brain, behaviour and cognition. Nat Rev Neurosci 10: $434-445$.

Menon V, Uddin LQ. 2010. Saliency, switching, attention and control: a network model of insula function. Brain Struct Funct 214: 655-667.

Miller EK, Li L, Desimone R. 1991. A neural mechanism for working and recognition memory in inferior temporal cortex. Science 254: $1377-1379$.

Moritz S, Woodward TS. 2005. Jumping to conclusions in delusional and non-delusional schizophrenic patients. Br J Clin Psychol 44: 193-207.

Oitzl MS, de Kloet ER. 1992. Selective corticosteroid antagonists modulate specific aspects of spatial orientation learning. Behav Neurosci 106: 62-71.

Power JD, Barnes KA, Snyder AZ, Schlaggar BL, Petersen SE. 2012. Spurious but systematic correlations in functional connectivity MRI networks arise from subject motion. Neuroimage 59: 2142-2154.

Pruessner JC, Kirschbaum C, Meinlschmid G, Hellhammer DH. 2003. Two formulas for computation of the area under the curve represent measures of total hormone concentration versus time-dependent change. Psychoneuroendocrinology 28: 916-931.

Roediger HL, McDermott KB. 1995. Creating false memories: remembering words not presented in lists. J Exp Psychol Learn Mem Cogn 21: 803-814.

Roozendaal B, Hahn EL, Nathan SV, de Quervain DJ, McGaugh JL. 2004. Glucocorticoid effects on memory retrieval require concurrent noradrenergic activity in the hippocampus and basolateral amygdala. $J$ Neurosci 24: 8161-8169.

Roozendaal B, Okuda S, Van der Zee EA, McGaugh JL. 2006. Glucocorticoid enhancement of memory requires arousal-induced noradrenergic activation in the basolateral amygdala. Proc Natl Acad Sci 103: 6741-6746.

Roozendaal B, McEwen BS, Chattarji S. 2009. Stress, memory and the amygdala. Nat Rev Neurosci 10: 423-433.

Rumelhart DE. 1980. Schemata: the building blocks of cognition. In Theoretical issues in reading comprehension: perspectives from cognitive psychology, linguistics, artificial intelligence, and education (ed. RJ Spiro, WF Brewer). Lawrence Erlbaum, Hillsdale, NJ.

Sandi C, Haller J. 2015. Stress and the social brain: behavioural effects and neurobiological mechanisms. Nat Rev Neurosci 16: 290-304.

Schwabe L, Wolf OT. 2009. Stress prompts habit behavior in humans. J Neurosci 29: 7191-7198.

Schwabe L, Wolf OT. 2012. Stress modulates the engagement of multiple memory systems in classification learning. J Neurosci 32: 11042-11049.

Schwabe L, Bohringer A, Chatterjee M, Schachinger H. 2008. Effects of prelearning stress on memory for neutral, positive and negative words: different roles of cortisol and autonomic arousal. Neurobiol Learn Mem 90: $44-53$.

Schwabe L, Schächinger H, de Kloet ER, Oitzl MS. 2010. Corticosteroids operate as a switch between memory systems. J Cogn Neurosci 22: 1362-1372.

Schwabe L, Joëls M, Roozendaal B, Wolf OT, Oitzl MS. 2012a. Stress effects on memory: an update and integration. Neurosci Biobehav Rev 36: $1740-1749$.

Schwabe L, Tegenthoff M, Höffken O, Wolf OT. 2012b. Simultaneous glucocorticoid and noradrenergic activity disrupts the neural basis of goal-directed action in the human brain. J Neurosci 32: 10146-10155.
Steyer R, Schwenkmezger P, Notz P, Eid M. 1994. Testtheoretische analysen des mehrdimensionalen befindlichkeitsfragebogens (mdbf). Diagnostica 40: $320-328$.

Tse D, Langston RF, Kakeyama M, Bethus I, Spooner PA, Wood ER, Witter MP, Morris RGM. 2007. Schemas and memory consolidation. Science 316: 76-82.

Tse D, Takeuchi T, Kakeyama M, Kajii Y, Okuno H, Tohyama C, Bito H, Morris RG. 2011. Schema-dependent gene activation and memory encoding in neocortex. Science 333: 891-895.

van Buuren M, Kroes MCW, Wagner IC, Genzel L, Morris RGM, Fernández G. 2014. Initial investigation of the effects of an experimentally learned schema on spatial associative memory in humans. J Neurosci 34: 16662-16670.

Vandenberghe R, Price C, Wise R, Josephs O, Frackowiak RS. 1996. Functional anatomy of a common semantic system for words and pictures. Nature 383: 254-256.

Vanelst B, Huybrechts I, de Bourdeaudhuij I, Bammann K, Hadjigeorgiou C, Eiben G, Konstabel K, Michels N, Molnar D, Moreno LA, et al. 2012. Prevalence of negative life events and chronic adversities in European pre- and primary-school children: results from the IDEFICS study. Arch Public Health 70: 26.

van Kesteren MTR, Fernández G, Norris DG, Hermans EJ. 2010a. Persistent schema-dependent hippocampal-neocortical connectivity during memory encoding and postencoding rest in humans. Proc Natl Acad Sci 107: 7550-7555.

van Kesteren MTR, Rijpkema M, Ruiter DJ, Fernández G. 2010b. Retrieval of associative information congruent with prior knowledge is related to increased medial prefrontal activity and connectivity. J Neurosci 30: $15888-15894$.

van Kesteren MTR, Ruiter DJ, Fernández G, Henson RN. 2012. How schema and novelty augment memory formation. Trends Neurosci 35: 211-219.

van Kesteren MTR, Beul SF, Takashima A, Henson RN, Ruiter DJ, Fernández G. 2013. Differential roles for medial prefrontal and medial temporal cortices in schema-dependent encoding: from congruent to incongruent. Neuropsychologia 51: 2352-2359.

van Kesteren MTR, Rijpkema M, Ruiter DJ, Morris RGM, Fernández G. 2014 Building on prior knowledge: schema-dependent encoding processes relate to academic performance. J Cogn Neurosci 26: 2250-2261.

Vogel S, Schwabe L. 2016. Stress in the zoo: tracking the impact of stress on memory formation over time. Psychoneuroendocrinology 71: 64-72.

Vogel S, Fernandez G, Joëls M, Schwabe L. 2016. Cognitive adaptation under stress: a case for the mineralocorticoid receptor. Trends Cogn Sci 20: 192-203.

Wagner IC, van Buuren M, Kroes MC, Gutteling TP, van der Linden M, Morris RG, Fernandez G. 2015. Schematic memory components converge within angular gyrus during retrieval. Elife 4: e09668.

Wang SH, Morris RGM. 2010. Hippocampal-neocortical interactions in memory formation, consolidation, and reconsolidation. Annu Rev Psychol 61: 49-79.

Wiegert O, Joels M, Krugers HJ. 2006. Timing is essential for rapid effects of corticosterone on synaptic potentiation in the mouse hippocampus. Learn Mem 13: 110-113.

Zoladz PR, Clark B, Warnecke A, Smith L, Tabar J, Talbot JN. 2011. Prelearning stress differentially affects long-term memory for emotional words, depending on temporal proximity to the learning experience. Physiol Behav 103: 467-476.

Received June 6, 2017; accepted in revised form September 26, 2017. 


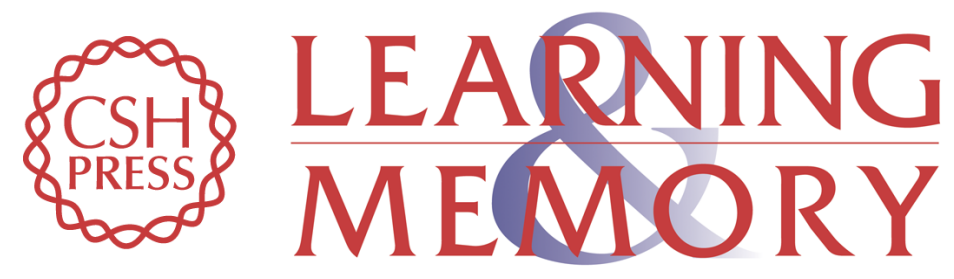

\section{Stress leads to aberrant hippocampal involvement when processing schema-related information}

Susanne Vogel, Lisa Marieke Kluen, Guillén Fernández, et al.

Learn. Mem. 2018, 25:

Access the most recent version at doi:10.1101/Im.046003.117

\section{Supplemental http://learnmem.cshlp.org/content/suppl/2017/12/08/25.1.21.DC1 Material}

References This article cites 62 articles, 17 of which can be accessed free at: http://learnmem.cshlp.org/content/25/1/21.full.html\#ref-list-1

Creative This article is distributed exclusively by Cold Spring Harbor Laboratory Press for the Commons first 12 months after the full-issue publication date (see

License http://learnmem.cshlp.org/site/misc/terms.xhtml). After 12 months, it is available under a Creative Commons License (Attribution-NonCommercial 4.0 International), as described at http://creativecommons.org/licenses/by-nc/4.0/.

Email Alerting Receive free email alerts when new articles cite this article - sign up in the box at the Service top right corner of the article or click here. 